\title{
Trasplante pulmonar en Uruguay. Avances en curso para la consolidación del programa
}

\author{
Nicolás Tommasino*, Cecilia Chao ${ }^{\dagger}$, Ana Musettił, Alejandro Bertolotti§, \\ Paola Lattanzio", Jorge Caneva§, Pablo Dibarboureף, Juan Manuel Osses§, \\ Guillermo Carriquiry ${ }^{\star *}$, Pablo Curbelot†
}

\section{Resumen}

Introducción: en Uruguay funciona desde el año 2003 un programa de trasplante pulmonar (TP) mediante un convenio binacional con la República Argentina, con Fundación Favaloro, centro regional de referencia.

Objetivos: describir los resultados del programa y herramientas en curso para mejorar la procuración pulmonar en Uruguay. Material y método: estudio descriptivo, retrospectivo, entre 2003 y 2017. Recopilación de datos del registro electrónico y análisis mediante SPSS.

Resultados: ingresaron a lista 70 pacientes, 27 fueron trasplantados, 95\% se reinsertaron a actividades sociales o laborales. Las etiologías fueron enfisema (33\%), fibrosis quística (26\%) y fibrosis pulmonar idiopática (11\%). Las complicaciones más frecuentes fueron infecciones respiratorias y alteraciones de la vía aérea. La mortalidad postrasplante es $34 \%$ y la supervivencia mediana condicional supera los ocho años. La elevada mortalidad en lista (32\%) impulsó el establecimiento de nuevas estrategias de procuración pulmonar. Destacamos: seguimiento longitudinal de pacientes, modificación de umbral de convocatoria, realización de maniobras de reclutamiento alveolar, realización sistemática de fibrobroncoscopía; minimización de tiempos de traslado y entrenamiento de equipo quirúrgico uruguayo para ablación. La procuración pulmonar pasó de 0 por millón de población (pmp) en 2014 y 2015 a 1,8 pmp en 2017.

Conclusiones: el Programa Uruguayo de Trasplante Pulmonar ha tenido importantes avances. Los últimos dos años han sido claves para el crecimiento de la procuración pulmonar. Los resultados, la sobrevida y la morbimortalidad son comparables a los descritos internacionalmente. Las perspectivas a futuro serán consolidar el programa en un centro de referencia y realización de ablación e implante en nuestro territorio.

Palabras clave: Trasplante de pulmón

Uruguay

Key words: $\quad$ Lung transplantation

Argentina

Uruguay

Selección de paciente

Argentina

Patient selection

\footnotetext{
* Asistente del Instituto Nacional de Donación y Trasplante.

† Asistente de Neumología, Instituto del Tórax, Hospital Maciel.

¥ Profesor Agregado de Neumología, Cátedra de Neumología, Hospital de Clínicas.

$\S$ Coordinador de Trasplante Pulmonar, Hospital Universitario. Fundación Favaloro.

ๆ Asistente de Cirugía de Tórax. Instituto del Tórax. Hospital Maciel.

** Profesor de Cirugía de Tórax. Instituto del Tórax. Hospital Maciel.

†† Profesor de Neumología. Coordinador del Programa de Trasplante Pulmonar. Instituto del Tórax, Hospital Maciel.

Correspondencia: Cecilia Chao. Correo electrónico: cecichp@gmail.com

Los autores declaran no tener conflicto de interés.

Recibido: $27 / 8 / 18$

Aprobado: 19/12/18
} 


\section{Introducción}

El trasplante pulmonar (TP) es una terapéutica válida en enfermedades respiratorias crónicas que no responden a pesar del tratamiento médico o quirúrgico optimizados, cuya expectativa de vida es muy reducida ${ }^{(1)}$. En estas enfermedades suele ser la única intervención capaz de modificar el pronóstico, la supervivencia y la calidad de vida. El TP se ha consolidado en los últimos 30 años; el número de trasplantes realizados tiende a crecer cada año, pasando de unos 15 por año realizados a mediados de la década de 1980 a 4.122 reportados en el mundo durante el $2015^{(2)}$.

En América Latina los resultados distan notablemente de ser óptimos y la tasa de donantes por millón de población (pmp) se encuentra lejos de las posibilidades máximas de la región. A modo de ejemplo, mientras que en América Latina, con 531,1 millones de habitantes, en el año 2016 se realizaron 167 TP, en Europa, con una población menor (505,9 millones) se realizaron 1.916, casi 11 veces más ${ }^{(3)}$. La escasez relativa de donantes atenta contra la formación de programas específicos. Actualmente, los únicos países que han reportado datos de TP realizados en América Latina en 2016 han sido Argentina, Brasil, Chile, Perú, Costa Rica y Uruguay ${ }^{(3)}$.

En nuestro país, con un bajo número de habitantes, se ha optado hasta el momento por un programa de trasplante en colaboración con un centro regional de referencia en TP (Fundación Favaloro para la Docencia e Investigación Médica, Buenos Aires, Argentina), mediante un convenio binacional con dicha República.

Los objetivos del presente trabajo son describir las características del programa de TP en Uruguay, mostrar sus resultados y avances, así como las herramientas en curso para mejorar la procuración pulmonar y aspectos operativos del mismo.

\section{Material y método}

Trabajo descriptivo de los antecedentes y situación actual del programa, así como de los avances en curso. Para la sección Resultados se realizó revisión retrospectiva de historias clínicas y análisis de base de datos del programa. El análisis estadístico se realizó mediante IBM SPSS Statistics v.20.

\section{Características del programa}

El programa funciona desde el año 2003 mediante un convenio binacional con la República Argentina a través de sus organismos reguladores INCUCAI (Instituto Nacional Central Único Coordinador de Ablación e Implante, Argentina) e INDT (Instituto Nacional de Donación y Trasplante, Uruguay) ${ }^{(4)}$.

En el marco de este convenio, en Uruguay se realizan los módulos de evaluación pretrasplante y segui- miento postrasplante. La ablación se realiza en territorio uruguayo por parte de un equipo quirúrgico proveniente de Fundación Favaloro que también realiza el implante en territorio argentino.

Este convenio establece que los pacientes uruguayos en situación de espera para trasplante electivo o de urgencia se trasplantarán con órganos procurados en nuestro país, mientras que en condición de emergencia los pacientes ingresan a lista argentina para trasplantarse con órganos procurados en el país vecino. Cada vez que esto último sucede, el INDT, en atención al principio de reciprocidad, se compromete a enviar al INCUCAI un órgano equivalente procurado en Uruguay con el objetivo de contribuir al programa de trasplante del cual se hizo usufructo.

El convenio binacional ha permitido el desarrollo y crecimiento de dicho programa en Uruguay gracias a la solidaridad y el trabajo en conjunto con los colegas argentinos.

El entrenamiento de recursos humanos ha sido continuo. Se progresó en el entrenamiento del equipo médico quirúrgico con pasantías de los cirujanos de tórax en uno de los principales centros europeos de referencia en TP, como lo es la Universidad de Lovaina. El equipo médico neumológico ha realizado su entrenamiento en forma permanente desde el año 2004 en el centro de referencia regional Hospital Universitario Fundación Favaloro.

El desarrollo del programa, necesario para abordar la alta demanda médica sanitaria dado el crecimiento sostenido de los potenciales receptores y la elevada mortalidad en lista de espera, presenta limitantes.

Las principales limitantes asociadas a la logística de los operativos de donación e implante son multicausales e incluyen tiempos prolongados del operativo, coordinación de la participación de técnicos de ambos países, coordinación de operativos de donación multiorgánica y otros imponderables, como la situación climática para el traslado o disponibilidad de aeropuertos durante las 24 horas.

Otra limitante no menor del programa es la falta de centralización asistencial en un centro único nacional de referencia en TP. Dicha centralización, que se encuentra en proceso de resolución administrativa, resulta indispensable para racionalizar la asistencia, optimizar el uso de recursos humanos, recursos tecnológicos asociados y costos económicos relacionados con la práctica. El abordaje de pacientes de la mayor complejidad en la trasplantología, como el TP, debe realizarse necesariamente en un centro asistencial de referencia que asegure los insumos, la tecnología, la logística y los recursos humanos interdisciplinarios de las especialidades involucradas.

\section{Discusión y resultados del programa}

Entre el 2003 y 2017, el incremento de pacientes remitidos para evaluación ha sido exponencial, debido princi- 


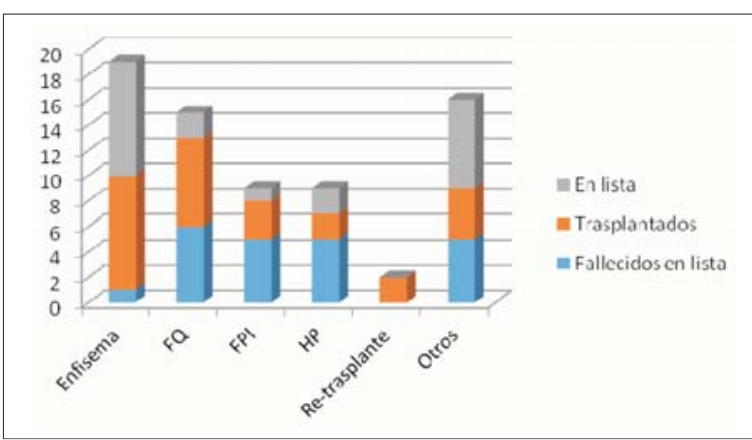

Figura 1. Evolución de los pacientes ingresados a lista.

palmente a la mayor difusión de esta posibilidad terapéutica y sus resultados entre los neumólogos del país. Durante los cuatro primeros años ingresaron a lista 15 pacientes ${ }^{(5)}$, mientras que considerando solo el 2017 ingresaron 9 pacientes. En total, han ingresado 70 pacientes a lista de espera desde el inicio del programa.

En cuanto a las características epidemiológicas y etiológicas de los pacientes ingresados en lista, las mismas se describen en la tabla 1. De los pacientes ingresados a lista, el 36\% se trasplantó, $4 \%$ se excluyó de lista, $2 \%$ requirió retrasplante, $32 \%$ falleció en lista y $26 \%$ se mantiene en lista. El enfisema y la fibrosis quística (FQ) fueron las causas más frecuentes de ingreso. El tiempo medio de espera en lista fue de 18,5 $\pm 18,24$ meses. La mortalidad global en lista fue elevada (32\%). Si analizamos la mortalidad según la patología, vemos que FQ, fibrosis pulmonar idiopática (FPI) y la hipertensión pulmonar (HP) fueron las de mayor mortalidad (figura 1).

En cuanto a la evolución del número de trasplantes por año, luego del primero en 2004, la cifra ha variado entre 1 y 3 al año, logrando un salto a seis trasplantes en 2017 (figura 2) gracias a estrategias en la procuración que luego detallaremos. En total, en los 14 años del programa se han realizado 27 trasplantes. De estos, más de $50 \%$ se han realizado en los últimos tres años, lo que muestra el cambio cuantitativo secundario a la aplicación de nuevas estrategias de procuración ${ }^{(6)}$. Las características de los pacientes trasplantados se detallan en la tabla 1. Predominan los trasplantes bipulmonares $(78 \%$ ) y fueron en condición electiva $56 \%$, en urgencia $11 \%$ y en emergencia $33 \%$.

Las patologías trasplantadas más frecuentes fueron enfisema, FQ y FPI. Esta distribución presenta grandes semejanzas con lo publicado internacionalmente, donde el enfisema también representa el $33 \%$ de los trasplantes, seguido por la enfermedad pulmonar intersticial (30\%), con predominio de la FPI $(24,8 \%)$, y en tercer lugar la enfermedad bronquiectásica FQ (16\%) y no FQ $(2,7 \%)^{(2)}$.

Las infecciones respiratorias han sido las complicaciones más frecuentes en nuestra casuística, seguidas de

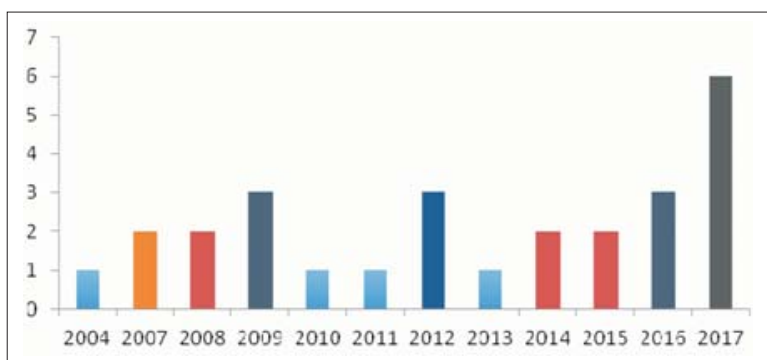

Figura 2. Número de trasplantes pulmonares por año.

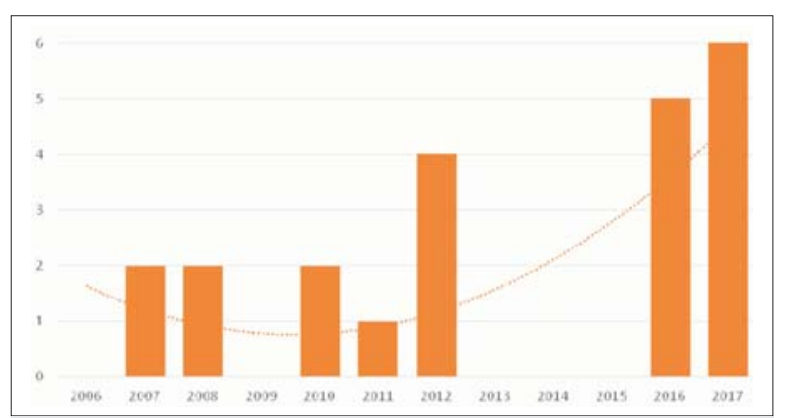

Figura 3. Procuración pulmonar por año.

las complicaciones de la vía aérea (33\% de los pacientes), en su mayoría a modo de estenosis bronquial.

En cuanto a los aspectos psicosociales, el $95 \%$ de los pacientes trasplantados lograron reinserción total a tareas laborales o educativas. Utilizando la escala Likert para la autovaloración de la calidad de vida (rango de 0 a 10), la media antes del trasplante se sitúa en 3,13 contrastando con una media de 9 postrasplante, reflejando el gran impacto positivo en los pacientes.

Hasta el momento analizado se trasplantaron 25 pacientes, dos se retrasplantaron. De los 25 pacientes, 18 se mantienen vivos (72\%). La mortalidad postrasplante se sitúa en $34 \%$, mientras que la mediana de supervivencia global y condicionada (excluyendo mortalidad del primer año postrasplante) por Kaplan-Meier coinciden en 8,48 años (figuras $4 a$ y $4 b$ ). Esta coincidencia responde al pequeño número de pacientes y la baja mortalidad (7 en 27). Según el reporte 2017 del Journal of Heart and Lung Transplantation (JHLT), la supervivencia media global del TP entre 1990 y 2016 ha sido de 6,0 años, y la supervivencia media condicional de 8,1 años. Con este análisis, podemos afirmar que nuestros resultados son comparables a los reportados internacionalmente ${ }^{(2)}$, si bien en nuestra serie existe el sesgo de ser un número escaso de pacientes. Debemos recordar que en las últimas tres décadas la supervivencia del TP se ha incrementado mundialmente, sobre todo a corto plazo, a expensas principalmente de las mejoras en el manejo perioperatorio de los pacientes, incluyendo la incorporación del 
Tabla 1. Características y etiologías de los pacientes ingresados a lista y trasplantados.

\begin{tabular}{|c|c|c|}
\hline$n$ & $\begin{array}{l}\text { Pacientes ingresados a lista } \\
70\end{array}$ & $\begin{array}{c}\text { Pacientes trasplantados } \\
27\end{array}$ \\
\hline Edad media (media $\pm \mathrm{DS}$ ) & $40,3 \pm 15,8$ & $39 \pm 16,8$ \\
\hline Sexo, Hombres (\%) & $37(53 \%)$ & $16(59 \%)$ \\
\hline \multirow[t]{9}{*}{ Etiologías } & Enfisema 27\% & Enfisema 33\% \\
\hline & $\mathrm{FQ} 21 \%$ & FQ 26\%. \\
\hline & FPI 13\% & FPI $11 \%$ \\
\hline & HP $13 \%$ & HP $7,4 \%$ \\
\hline & Otras EPI 16\% & BE no FQ $7,4 \%$ \\
\hline & BE no FQ 3\% & Re trasplante $7,4 \%$. \\
\hline & Re trasplante $3 \%$. & BO 3,9\% \\
\hline & BO $3 \%$ & Otras EPI $3,9 \%$ \\
\hline & Enfisema-fibrosis $1 \%$ & \\
\hline
\end{tabular}

FQ: fibrosis quística; FPI: fibrosis pulmonar idiopática; HP: hipertensión pulmonar; EPI: enfermedades pulmonares intersticiales; BE no FQ: bronquiectasias no fibrosis quística; BO: bronquiolitis obliterante.

soporte vital avanzado con el uso de la membrana de oxigenación extracorpórea (ECMO).

Respecto a las causas de muerte, dos pacientes fallecieron en el postrasplante inmediato (disfunción primaria severa del injerto y lesión vascular intraoperatoria), un paciente falleció por disfunción crónica del injerto $(\mathrm{CLAD})^{(6)}$ y los cuatro restantes por causa infecciosa, constituyendo la principal causa de muerte de nuestra serie. Dentro de los microorganismos involucrados, destacamos el aislamiento de Scedosporium en dos de ellos, Pseudomonas aeruginosa, Staphylococcus aureus, herpes virus y virus influenza $\mathrm{A}$.

Según la literatura, a pesar de la mejoría en los números globales de supervivencia, las causas de muerte no han variado significativamente con el correr de los años ${ }^{(8,9)}$. Las principales causas de muerte en el primer mes postrasplante son la disfunción primaria del injerto y las infecciones respiratorias, si bien contribuyen las complicaciones quirúrgicas y las causas cardiovasculares. Si consideramos el primer año, la principal causa de muerte se centra en las infecciones. Finalmente, pasado el primer año, cobra importancia el rechazo crónico $^{(7)}$, principalmente bajo la forma de síndrome de bronquiolitis obliterante $(\mathrm{SBO} / \mathrm{BOS}$, bronchiolitis obliterans syndrome), acompañado de infecciones. Aún dentro del primer año las enfermedades neoplásicas contribuyen en buen número a las causas de muerte ${ }^{(9)}$.

\section{Estrategias para incrementar el número de donantes pulmonares}

En todos los programas de TP el crecimiento de pacientes ingresados a lista de espera es desproporcionadamente alto respecto al número de trasplantes. Este disbalance producido por la elevada demanda y la escasez relativa de donantes es el principal responsable de la excesiva mortalidad en lista de espera. La escasez relativa de donantes es la mayor limitante para el desarrollo y la aplicación generalizada de esta terapéutica ${ }^{(10)}$.

En Uruguay, como se mencionó previamente, desde el inicio del programa hasta el año 2015, se realizaron 13 trasplantes con una media anual que oscilaba entre 0 y 2 , realizándose excepcionalmente cuatro trasplantes en el año 2012. Entre los años 2013 y 2015 el número de donantes pulmonares ablacionados en el país fue cero, lo que obligó al equipo de trasplante pulmonar, junto al INDT, a diseñar nuevas estrategias de procuración pulmonar.

Como primera medida, durante el 2015, se realizó un seguimiento prospectivo de todos los donantes reales menores de 65 años en quienes se analizaron las causas de descarte para pulmón. Se detectó que las principales causas de descarte fueron: tiempos de procuración previstos excesivamente largos (logística), el planteo no confirmado de aspiración del donante y el deterioro del intercambio gaseoso expresado por la relación $\mathrm{PaO}_{2} / \mathrm{FiO}_{2}$. Estas tres causas se asociaron a más del $60 \%$ 


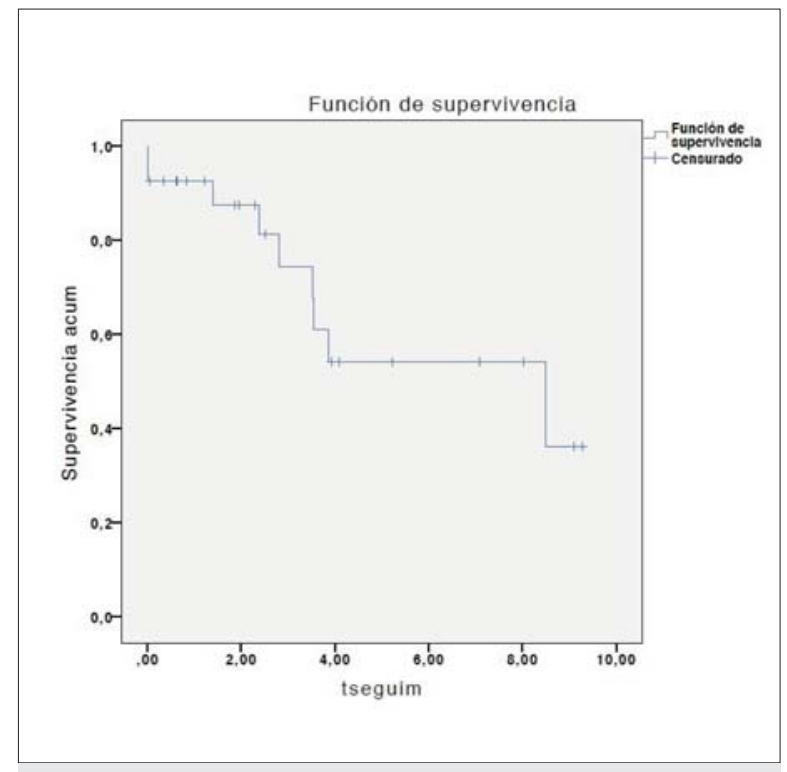

Figura 4a. Mediana de supervivencia Kaplan-Meier.

de los pulmones descartados. Las restantes eran causas no corregibles de descarte (contraindicaciones absolutas).

Se establecieron entonces las siguientes estrategias:

1. Se implementó un programa de seguimiento prospectivo de pacientes menores de 65 años comunicados al INDT como pasibles de evolucionar a muerte encefálica con el objetivo de minimizar el escape de donantes pulmonares mediante seguimiento longitudinal.

2. Se descendió el umbral de convocatoria al equipo de evaluación pulmonar a $\mathrm{PaO}_{2} / \mathrm{FiO}_{2}$ de $200 \mathrm{mmHg}$ con una PEEP hasta $8 \mathrm{~cm} \mathrm{H}_{2} \mathrm{O}$. En aquellos pacientes con $\mathrm{PaO}_{2} / \mathrm{FiO}_{2}$ aceptable en gasometrías arteriales previas y un deterioro de la $\mathrm{PaO}_{2} / \mathrm{FiO}_{2}$ posterior a la prueba de apnea se planteó la realización de maniobras de homogeneización pulmonar mediante PEEP para minimizar la participación de posibles atelectasias vinculadas a los períodos de desconexión de la asistencia respiratoria mecánica (ARM). En Uruguay son necesarios dos tests de apnea para el diagnóstico de muerte encefálica. Estos habitualmente se realizan sin válvula de PEEP y desconectado de la ARM, lo que deteriora el intercambio gaseoso. La realización de maniobras de reclutamiento alveolar ha demostrado incrementar el número de pulmones válidos para trasplante ${ }^{(11)}$.

3. Se generalizó la realización de fibrobroncoscopía en el potencial donante pulmonar procurando confirmar o descartar el planteo clínico de aspiración. Destacamos que no en todos los pacientes en los que se

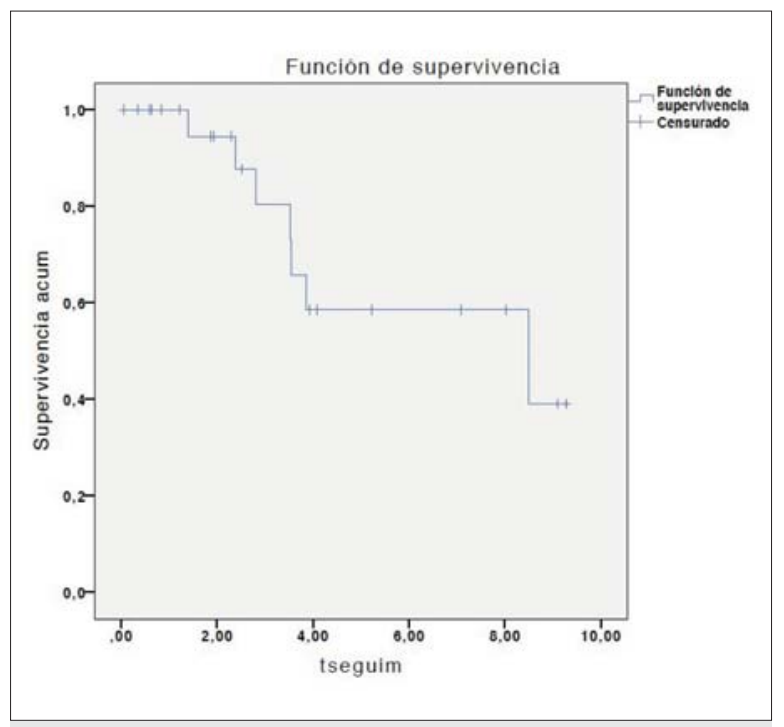

Figura 4b. Mediana de supervivencia condicional Kaplan-Meier.

planteó clínicamente aspiración, se confirmó por broncoscopía.

4. En cuanto a los aspectos logísticos se describieron las diferentes fases de los procesos de ablación, traslado del receptor y del órgano para implante. Se minimizaron los tiempos, independizando los traslados del receptor y del equipo de ablación.

5. Conformación de un equipo quirúrgico uruguayo, actualmente en entrenamiento, con el objetivo de contar en un futuro próximo con un equipo de ablación y posterior implante en nuestro territorio. Se comenzó el entrenamiento mediante la ablación pulmonar en donantes no óptimos rechazados como donantes reales y asimismo la realización de ablaciones en donantes óptimos por el equipo quirúrgico uruguayo supervisado por el equipo argentino. Esta incorporación tiene un doble propósito: realizar la curva de aprendizaje de ablación pulmonar y minimizar el escape de donantes pulmonares al generar un segundo control mediante el análisis macroscópico y gasométrico intraoperatorio ${ }^{(12,13)}$. Asimismo, se generó un protocolo de investigación que correlaciona los hallazgos clínicos, gasométricos, microbiológicos, anatomopatológicos y fisiopatológicos de los pulmones rechazados. Creemos que este protocolo en el futuro ayudará a la toma de decisiones en cuanto al rechazo de donantes pulmonares. Ya en los primeros operativos bajo esta modalidad hemos detectado discordancias en gasometrías realizadas mediante punción de arteria radial y gasometrías realizadas en venas pulmonares a tórax abierto, hecho previamente reportado por otros grupos de traba- 
jo ${ }^{(14,15)}$. También hemos detectado la presencia de macrotrombos pulmonares en pacientes con baja probabilidad clínica de tromboembolismo pulmonar, hecho también reportado previamente por otros grupos de trabajo $^{(16)}$. Estos hallazgos, de ser confirmados, pueden abrir la puerta a futuros algoritmos de decisión en cuanto a terapéutica de donantes y rechazo o aceptación.

Una vez entrenado el equipo quirúrgico local y validada su experiencia por parte del equipo quirúrgico de referencia, se minimizarán aún más los tiempos operativos. Esto permitirá la valoración in situ de pulmones con criterios límites de aceptación a priori.

6. En busca de nuevas fuentes de donantes se encuentra en desarrollo un programa de donación pulmonar en asistolia en un hospital de referencia nacional (Hospital Maciel). Este programa se encuentra en fase de reevaluación y expansión a nuevos centros.

A raíz de estas nuevas estrategias, si bien queda mucho trabajo por realizar, ya se comienzan a visualizar algunos resultados esperanzadores: la procuración pulmonar en Uruguay pasó de 0 pmp durante los años 2013, 2014 y 2015 a 1,2 pmp en 2016, lo que la ubicó en el segundo lugar de América Latina después de Chile ${ }^{(3)}$, y 1,8 pmp en 2017 .

\section{Conclusiones y desafíos futuros}

El Programa Uruguayo de Trasplante Pulmonar, gracias al trabajo en conjunto entre el equipo técnico local $\mathrm{y}$ el del centro de referencia, ha tenido importantes avances desde su inicio en 2003. Los últimos dos años han sido claves para el crecimiento de la procuración pulmonar. Los resultados en los pacientes trasplantados en cuanto a características, morbimortalidad, calidad de vida y supervivencia son comparables con los descritos internacionalmente. Dentro de los desafíos a corto plazo, destacamos la posibilidad de consolidación del equipo quirúrgico de ablación uruguayo, así como centralizar la actividad de seguimiento pre y postrasplante en un centro asistencial de referencia. Esta centralización es imperiosa y es la única forma de abordar un tratamiento y seguimiento integral e inter y multidisciplinario a pacientes de gran complejidad en todas sus etapas. Asimismo y en forma simultánea se deberá realizar el entrenamiento del equipo multidisciplinario involucrado en los distintos aspectos asistenciales (anestesia, terapia intensiva, anatomía patológica, entre otros). Estos aspectos serán claves para poder pensar en un eventual programa independiente incluyendo la ablación y el manejo posoperatorio en nuestro país en un futuro próximo.

\section{Abstract}

Introduction: a program for Lung Transplant (LT) has been operative in Uruguay since 2003, by means of a bi-national agreement with Fundación Favaloro, a regional reference center in Argentina.

Method: descriptive, retrospective study performed between 2003 and 2017. Data in the electronic register was compiled and analyzed using SPSS (Statistical Package for the Social Sciences).

Results: 70 patients were entered in the list, 27 of which underwent transplant surgery, $95 \%$ of them resumed social like and/or work. Etiologies included emphysema (33\%), cystic fibrosis (26\%) and idiopathic pulmonary fibrosis $(11 \%)$. The most frequent complications were respiratory infections and alterations in the air way. Post-transplant mortality is $34 \%$ and conditional median survival is higher than 8 years. The increased mortality in the list $(32 \%)$ encouraged development of new strategies for lung procurement. The following facts are pointed out: longitudinal follow-up of patients, modification of the calling threshold, the performance of alveolar recruitments maneuvers, the systematic performance of fibrobroncoscopy, minimization of transport times and training of the Uruguayan surgical team for ablation. Lung procurement increased from 0 per million of population (pm) in 2014 to $1.8 \mathrm{pmp}$ in 2017.

Conclusions: the Uruguayan program for LT has evidenced significant progress. The last two years have been essential for the increase of lung procurement. Results, survival and morbi-mortality are comparable to those described globally. Future perspectives will focus on consolidating the program in a reference center and performing ablation and implants in our country.

\section{Resumo}

Introdução: desde 2003 funciona no Uruguai um programa de Transplante Pulmonar (TP) através de um convenio binacional com Argentina, com Fundación Favaloro, centro regional de referência.

Objetivos: descrever os resultados do programa e as ferramentas que estão sendo utilizadas para melhorar a procuração pulmonar no Uruguai.

Materiais e métodos: estudo descritivo, retrospectivo do período 2003-2017. Coleta de dados do registro eletrônico e análise com o programa SPSS.

Resultados: ingressaram à lista 70 pacientes; 27 foram transplantados dos quais $95 \%$ voltaram a atividades sociais e/ou de trabalho. As etiologias foram enfisema (33\%), fibrose cística (26\%) e fibrose pulmonar idiopática $(11 \%)$. As complicações mais frequentes foram as infecções respiratórias e alterações da via aérea. A mortalidade pós-transplante foi de $34 \%$ e a sobrevida media- 
na condicional foi superior a 8 anos. Dada la elevada mortalidade na lista de espera (32\%) novas estratégias de procuração pulmonar foram estabelecidas. Destacamos: seguimento longitudinal dos pacientes, modificação do umbral de convocatória, realização de manobras de recrutamento alveolar, realização sistemática de fibrobroncoscopia, minimização dos tempos de traslado e capacitação da equipe uruguaia de cirurgia em ablação. A procuração pulmonar passou de 0 por milhão de habitantes (pmp) em 2014 e 2015 a 1.8 pmp em 2017.

Conclusões: o Programa Uruguaio de TP mostrou importantes avanços. Os últimos 2 anos foram fundamentais para o crescimento da procuração pulmonar. Os resultados, a sobrevida e a morbimortalidade são comparáveis aos descritos na literatura internacional. As perspectivas para o futuro são consolidar o programa em um centro de referência e a realização de ablação e implante no nosso território.

\section{Bibliografía}

1. Román A, Ussetti P, Solé A, Zurbano F, Borro JM, Vaquero JM, et al. Guidelines for the selection of lung transplantation candidates. Arch Bronconeumol 2011; 47:303-9.

2. Chambers DC, Yusen RD, Cherikh WS, Goldfarb SB, Kucheryavaya AY, Khusch K, et al. The registry of the international society for heart and lung transplantation: thirty-fourth adult lung and heart-lung transplantation report-2017; focus theme: allograft ischemic time. J Heart Lung Transplant 2017; 36(10):1047-59.

3. Red/Consejo Iberoamericano de Donación y Trasplante. Newsletter Trasplante Iberoamérica 2017; XI(1). Disponible en: http://www.ont.es/publicaciones/Documents/NEWSLETTER \%202017_baja\%20(2).pdf [Consulta: 12 de junio de 2018].

4. Curbelo P, Cáneva J, Ossés JM, Bertolotti A, Álvarez I, Mizraji R, et al. Programa de Trasplante Pulmonar en Uruguay. Nuestra experiencia con un centro regional de referencia. Rev Argent Med Respir 2008; 8:103-6.

5. Curbelo P. Trasplante pulmonar: revisión. Situación en Uruguay. Arch Med Interna 2008; Supl 2:S44-8.

6. Uruguay. Ministerio de Salud Pública. Instituto Nacional de Donación y Trasplante de Células, Tejidos y Órganos. Estadísticas (Actualizadas a diciembre 2017). 2017. Disponi- ble en: https://www.indt.gub.uy/?S=estadisticas [Consulta: 5 de agosto de 2018]

7. Curbelo P, Cáneva J, Chao C, Ossés JM, Vigliano C, Tommasino N, et al. Fenotipos de disfunción crónica del injerto pulmonar. Rev Am Med Respir 2018; 2:121-4.

8. Christie JD, Edwards LB, Kucheryavaya AY, Aurora P, Dobbels F, Kirk R, et al. The registry of the International Society for Heart and Lung Transplantation: twenty-seventh official adult lung and heart-lung transplant report-2010. J Heart Lung Transplant 2010; 29:1104-18.

9. Trulock EP, Edwards LB, Taylor DO, Boucek MM, Keck BM, Hertz MI. Registry of the International Society for Heart and Lung Transplantation: twenty-second official adult lung and heart-lung transplant report-2005. J Heart Lung Transplant 2005; 24:956-67.

10. Yusen RD, Edwards LB, Kucheryavaya AY, Benden C, Dipchand AI, Dobbels F, et al. The registry of the International Society for Heart and Lung Transplantation: thirty-first adult lung and heart-lung transplant report-2014; focus theme: retransplantation. J Heart Lung Transplant 2014; 33:1009-24.

11. Miñambres E, Pérez-Villares JM, Chico-Fernández M, Zabalegui A, Dueñas-Jurado JM, Misis M, et al. Lung donor treatment protocol in brain dead-donors: a multicenter study. J Heart Lung Transplant 2015; 34:773-80.

12. Miñambres E, Coll E, Duerto J, Suberviola B, Mons R, Cifrian JM, et al. Effect of an intensive lung donor-management protocol on lung transplantation outcomes. J Heart Lung Transplant 2014; 33:178-84.

13. Martens A, Neyrinck A, Van Raemdonck D. Accepting donor lungs for transplant: let Lisa and Bob finish the job! Eur J Cardiothorac Surg 2016; 50:832-3.

14. Ware LB, Wang Y, Fang X, Warnock M, Sakuma T, Hall TS, et al. Assessment of lungs rejected for transplantation and implications for donor selection. Lancet 2002; 360(9333):619-20.

15. Costa J, Sreekanth S, Kossar A, Raza K, Lederer DJ, Robbins H, et al. Donor lung assessment using selective pulmonary vein gases. Eur J Cardiothorac Surg 2016; 50:826-31.

16. Ware LB, Fang X, Wang Y, Babcock WD, Jones K, Matthay MA. High prevalence of pulmonary arterial thrombi in donor lungs rejected for transplantation. J Heart Lung Transplant 2005; 24:1650-6. 